\title{
Buprenorphine Measurement
}

National Cancer Institute

\section{Source}

National Cancer Institute. Buprenorphine Measurement. NCI Thesaurus. Code C75352.

The determination of the amount of buprenorphine present in a sample. 